\title{
Cauda equina syndrome in an obese pregnant patient secondary to double level lumbar disc herniation - A case report and review of literature
}

\author{
Dilip Chand Raja S $\mathbb{D}^{1} \cdot$ Ajoy Prasad Shetty ${ }^{1} \cdot$ Rishi Mugesh Kanna ${ }^{1} \cdot S$ Rajasekaran ${ }^{1}$
}

Received: 8 January 2019 / Revised: 8 February 2019 / Accepted: 2 March 2019

(c) International Spinal Cord Society 2019

\begin{abstract}
Introduction Lumbar disc herniation during pregnancy poses a significant challenge to the spine surgeon towards achieving good clinical, maternal, and fetal outcomes. Surgical intervention is warranted in patients with significant neural deficits, and cauda equina syndrome and needs to be performed at the earliest in order to avoid irreversible neurological sequelae.

Case presentation We report a 29-year-old primigravida in her 21st week of gestational period, who was diagnosed with cauda equina syndrome secondary to two level lumbar disc herniations. The lengthier surgical duration in performing double level disc herniations in an obese patient raises concerns in anesthetic dosing of drugs and surgical positioning which may result in fetal distress. A double level decompression and discectomy in prone position was done under general anesthesia. Despite the surgical challenges, the postoperative period was uneventful resulting in immediate pain relief and complete neurological recovery, followed by the delivery of a $2.7-\mathrm{kg}$ healthy male child.

Discussion Surgical intervention can be performed in pregnancy, to avoid irreversible neurological deficits, even in an obese individual with double level lumbar pathology. However, it is essential that the surgeon appraises the situation and involves an integrated multidisciplinary team comprising anesthetist, spine surgeon, obstetrician, and psychologist, and inculcates certain precautions in the perioperative management to achieve good surgical and fetal outcomes.
\end{abstract}

\section{Introduction}

Despite low back pain being the most common musculosketal disorder in pregnancy, the incidence of lumbar disc herniations is only 1 in 10,000 and that of cauda equina syndrome is even more rare [1]. A high index of suspicion is essential in diagnosing cauda equina syndrome due to the masquerading pregnancy associated bowel and bladder disturbances [2]. There is evidence to suggest the safety of surgical intervention to address the incapacitating pain and neurological disturbances secondary to single-level disc herniation in pregnancy [3]. However, the lengthier surgical duration in performing double level disc herniations in an obese patient raises concerns in anesthetic dosing of drugs and surgical positioning which may result in fetal distress.

Ajoy Prasad Shetty

ajoyshetty@gmail.com

1 Department of Spine Surgery, Ganga Hospital, 313, Mettupalayam road, Coimbatore, India
We report here the successful surgical management of two level disc herniations in an obese primiparous woman at 21 weeks of gestation and discuss pertinent literature on surgical considerations in pregnancy.

\section{Case presentation}

A 29-year-old primigravida presented to the emergency department at 21 st week of gestation with history of severe low back pain for 4 weeks associated with excruciating right lower limb radiculopathy since 2 weeks. On examination, she had complete weakness of right extensor hallucis longus and tibialis anterior (MRC grade-0). Hypoesthesia of right L4 and L5 sensory dermatomes, saddle anesthesia along with nerve root tension signs were suggestive of cauda equina syndrome. Positioning this patient for elicitation of Bulbocavernosus reflex was difficult because of pregnancy and obesity. Voluntary anal contraction was present though weak and post voidal painless urinary retention of $850 \mathrm{ml}$ confirmed the diagnosis of cauda equina syndrome. Magnetic Resonance Imaging 
(MRI) (Fig. 1) revealed L3-L4 and L4-L5 disc herniations with severe canal and foraminal stenosis, consistent with the clinical neurological weakness. The cross-sectional area of thecal sac was reduced to $24.58 \mathrm{~mm}^{2}$ at L3-L4 and $61.24 \mathrm{~mm}^{2}$ at L4-L5 level. She was weighing $110 \mathrm{~kg}$ with a BMI of 37.7 at presentation. A multidisciplinary team comprising an obstetrician, anesthesiologist, spine surgeon, and psychologist was involved and the necessity of surgical intervention to prevent irreversible neurological deficits was explained. All maternal and fetal risk factors associated with surgery in pregnancy were discussed in depth and after consent surgery was planned.

Preoperatively, she was started on subcutaneous dose of dalteparin sodium (5000 IU) and was continued for 1 week. In addition, single dose of intramuscular hydroxyprogesterone caproate $(500 \mathrm{mg})$ was used to lower the risk of miscarriage. With standard monitoring devices, induction of general anesthesia was done with Fentanyl $2 \mu \mathrm{g} / \mathrm{kg}$ and Propofol $2 \mathrm{mg} / \mathrm{kg}$ after pre-oxygenation with $100 \%$ oxygen for $5 \mathrm{~min}$. Tracheal intubation was facilitated by administering Rocuronium $0.8 \mathrm{mg} / \mathrm{kg}$. Antibiotic prophylaxis was initiated with $1.5 \mathrm{gm}$ of cefuroxime half hour before surgery and was followed by two additional doses postoperatively. One gram of paracetamol infusion and $8 \mathrm{mg}$ Dexamethasone IV were administered after skin incision. Patients' lungs were ventilated with a tidal volume of $7-9 \mathrm{~mL} / \mathrm{kg}$, I: E ratio of $1: 2$ at a respiratory rate of $8-12$ breaths/min in $50 \%$ oxygen and $\mathrm{N} 2 \mathrm{O}$ to maintain normocarbia $(\mathrm{aPco} 2=30-40 \mathrm{~mm} \mathrm{Hg}$ ) throughout the surgery. Anesthesia was maintained with continuous inhalational agent desflurane (1 MAC) and top up doses of Rocuronium at $0.1 \mathrm{mg} / \mathrm{kg}$ were used. The patient was positioned prone on high bolsters with adequate padding of bony prominences with due care to prevent aortocaval compression and fetal distress. Pneumatic compression devices were used in the intra and post-operative period. Maternal hemodynamic parameters and oxygenation were carefully monitored throughout the surgery. Microscopic decompression and discectomy was done at L3-L4 level along with L4-L5 discectomy. Reversal of general anesthesia was performed with neostigmine $(50 \mu \mathrm{g} / \mathrm{kg})$ and glycopyrrolate $(10 \mu \mathrm{g} / \mathrm{kg})$. After surgery, fetal monitoring was done by ultrasound and there was no evidence of fetal distress. Postoperative surgical pain was managed with parenteral infusion of $1 \mathrm{gm}$ paracetamol. The patient had immediate pain relief following surgery and the neurological deficit recovery was gradual and complete by 4 months. At 39 weeks of gestation, considering the two level disc degeneration and severe obesity, she was planned for elective cesarean section and delivered a healthy $2.7 \mathrm{~kg}$ male child. It has been 2 years since surgery and there is no evidence of recurrent pathology with both mother and child in good health.

\section{Discussion}

Low back pain is common amongst pregnant women and affects nearly $50 \%$ of individuals and several hypothesis have been proposed behind its etiology. Exaggerated lordosis, intrauterine weight, laxity of abdominal muscles resulting in increased intradiscal pressure and stretch of ligamentous structures due to production of relaxin in the third trimester, contribute to low back pain during pregnancy [4]. However, the incidence of symptomatic disc herniations during pregnancy is only 1 in 10000 . Cauda
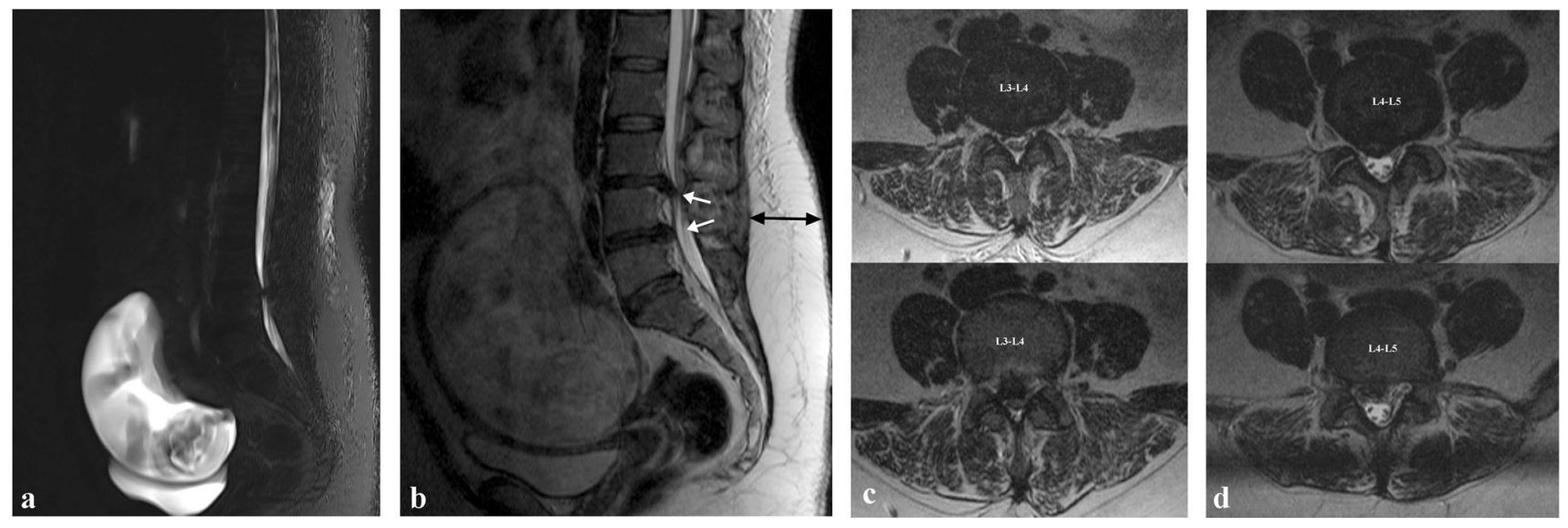

Fig. 1 a Myelographic sequence in MRI demonstrating an intrauterine gestation of 21 weeks and severe cauda equina compression in a 29year-old obese primiparous mother. b T2 weighted sagittal sequence with white arrows pointing at L3-L4 and L4-L5 disc herniations and black arrow demonstrating the huge amount of subcutaneous fat adding to the surgical challenge in addressing the two level pathology with neurodeficit in this obese mother during second trimester of pregnancy. c Axial T2 images at L3-L4 showing a right posterolateral disc extrusion with inferior migration causing severe compression. d Axial image at L4-L5 showing a huge L4-L5 disc extrusion with inferior migration extending up to the pedicle level 
equina syndrome is an even more rare entity affecting only $2-6 \%$ of all disc herniations [5]. Clinical examination of subtle neurological disturbances is of paramount importance in diagnosing cauda equina syndrome. Subtle bowel and bladder disturbances might be overlooked during pregnancy leading to delayed diagnosis and irreversible neurological deficits [6]. MRI is safe and is the radiological investigation of choice in pregnancy and provides excellent aid in early diagnosis [7]. However, MRI with contrast agents are to be avoided as they have been proven to induce spontaneous abortions in animal studies. Surgical intervention within $48 \mathrm{~h}$ of onset of symptoms is crucial and carries the best prognosis.

There is strong evidence that surgery for disk herniation is safe in pregnancy [8]. However, the surgeon should contemplate on the choice of anesthesia, surgical positioning, and perioperative care to avoid maternal or fetal distress. Prone positioning reduces epidural venous pressure and provides excellent posterior access to a herniated disc and is safe in first and second trimester. However, in third trimester, prone positioning with a gravid uterus may cause aortocaval compression resulting in fetal distress. Henceforth, lateral positioning is generally preferred. In patients near term, performing an elective cesarean in supine position followed by posterior surgery in prone position is an option [9]. In our case, we had to perform discectomy at two levels in a severe obese, pregnant lady, in second trimester. While discectomy at L3-L4 alone could be a surgical option, there was a significant right sided disc extrusion at L4-L5 level and therefore right sided radiculopathy could not be acclaimed only to the L3-L4 level disc prolapse. Moreover, the probability of persistent right radiculopathy was not acceptable to the patient and the relatives, and therefore they preferred to undergo surgery at two levels. We used high bolsters with additional padding instead of the routine Relton hall frame considering a longer duration of surgery to address two levels rather than a single level surgery in other cases reported. The total duration of surgery was $94 \mathrm{~min}$ in our case. The use of intraoperative versus postoperative fetal heart beat monitoring is a matter of debate. Overall, there is no need of monitoring until 20 weeks of gestation, its use beyond 23 week is mandatory and between 20 and 23 weeks, its use is questionable. In our case there was good cardiac activity at 21 st week of gestation and was therefore monitored, immediately after surgery.

Regional anesthesia is believed to be the safest as there is a theoretical risk of difficult airway aspiration to the mother in general anesthesia. In addition, there is potential of drug exposure to fetus, low birth weight, and chances of preterm labor. However, there are no controlled trials to prove the ill effects of general anesthesia and it has been successfully used with no additional complications. More recently, percutaneous endoscopic lumbar discectomy in lateral decubitus has been claimed to have an additional advantage of avoiding general anesthesia. However, the need for fluoroscopy, longer learning curve, and lengthier duration of surgery are factors to be contemplated [10]. The mean arterial pressure should be maintained at $60 \mathrm{~mm} \mathrm{Hg}$ and thromboembolic prophylaxis during the perioperative period is vital. Pain management should be given special care. Whilst, paracetamol and cyclobenzaprine are considered safe during pregnancy, opioids and steroids are known to be teratogenic in the first trimester and are best avoided. Though Nonsteroidal anti-inflammatory drugs (NSAIDS) are safe, its use in third trimester might cause premature closure of patent ductus arteriosus. We had used only parenteral paracetamol infusion for pain management and changed to oral paracetamol on third post-operative period. The patient had excellent relief of pain following surgery. Motor recovery of Tibialis anterior and Extensor hallucis Longus was gradual and improved from Grade 0/5 preoperatively to Grade 3/5 at first month of follow up and recovered completely at four months following surgery. Considering the huge preoperative post voidal urinary retention, she was catheterized for a period of one week and then checked for recovery of bladder symptoms. Due to persistent high volume of urinary retention $(550 \mathrm{ml})$ she was put on a tailored rehabilitation program for bladder training, where she required clean intermittent self-catheterization for a period of 1 month before returning to normal bladder habits. Fortunately, though she had constipation for a period of two weeks, she had no fecal incontinence. The antenatal progress was normal and elective cesarean section was performed at 39 weeks of gestation. A healthy $2.7 \mathrm{Kg}$ heathy male child was delivered. It is now 2 years since surgery and both the child and mother are in good health.

Differential diagnosis of low back pain during pregnancy cannot be overemphasized as failure in early diagnosis might lead to unnecessary neurological sequelae which not only influences maternal and fetal outcomes but also affects parenting. Recently, a pregnant woman had to undergo an urgent thoracic laminectomy, due to progressive weakness following a missed diagnosis of spinal Ependymoma which presented as thoracic back pain as early as 8 weeks of gestation [11]. However, the signs were attributed towards normal pregnancy related pain by a primary care physician and a chiropractor and she worsened over 10 weeks when an MRI revealed a spinal tumor. She was by then having significant neurological weakness and was not ambulating on her own. The situation in a paraplegic patient due to thoracic level pathology is even more worse considering the altered bowel and bladder habits, Autonomic dysreflexia, inability to feel uterine movements and monitor fetal status, higher risks for breech/transverse fetal positioning, and difficulty in initiation of breast feeding. Occupational, 
Physical and Psychological rehabilitation by a well-trained spinal cord disorder team in collaboration with the obstetricians is very essential in improving the functional outcomes of these patients. More importantly, the physicians need to be aware of such clinical scenarios in order to diagnose these patients appropriately and also engage an interdisciplinary team in the management of these patients.

\section{Conclusion}

Cauda equina syndrome is a surgical emergency and there should be no reason for delay in intervention even in a pregnant patient. There is a risk of missing early bowel and bladder involvement in pregnancy due to overlapping symptoms and therefore a high index of suspicion is essential in such cases when associated with back pain and radiculopathy. To our knowledge, this case represents the first case of double level discectomy in a pregnant patient with cauda equina syndrome. Though surgical intervention is safe during pregnancy, it is essential that the surgical team appraises the situation and inculcates certain precautions in the perioperative management to achieve good surgical and fetal outcomes.

Acknowledgements We contributed equally towards the preparation of this manuscript and we acknowledge all of their efforts.

\section{Compliance with ethical standards}

Conflict of interest The authors declare that they have no conflict of interest.
Publisher's note: Springer Nature remains neutral with regard to jurisdictional claims in published maps and institutional affiliations.

\section{References}

1. Han IH. Pregnancy and spinal problems. Curr Opin Obstet Gynecol. 2010;22:477-81.

2. Mohapatra RN, Patra RK. Cauda equina syndrome in pregnancy due to disc prolapse. J, Indian Acad Clin Med. 2008;9:141.

3. Fahy UM. Surgical management of herniated lumbar disc in pregnancy. J Obstet Gynaecol. 1998;18:544-5.

4. Kelsey JL, Greenberg RA, Hardy RJ, Johnson MF. Pregnancy and the syndrome of herniated lumbar intervertebral disc; an epidemiological study. Yale J Biol Med. 1975;48:361.

5. Pinheiro JF, Hernández DP, Blanco JF. Cauda equina syndrome during pregnancy: a condition to consider. Int J Surg Case Rep. 2018;49:14-16.

6. Kanas M, Kunzle H, Martins DE, Kirsch LA, Puertas EB, Wajchenberg M. Diskectomy during pregnancy: case report and review of the literature. Glob Spine J. 2015;5:130-4.

7. LaBan MM, Rapp NS, von Oeyen P, Meerschaert JR. The lumbar herniated disk of pregnancy: a report of six cases identified by magnetic resonance imaging. Arch Phys Med Rehabil. 1995;76:476-9.

8. Di Martino A, Russo F, Denaro L, Denaro V. How to treat lumbar disc herniation in pregnancy? A systematic review on current standards. Eur Spine J. 2017;26:496-504.

9. Ochi H, Ohno R, Kubota M, Hanyu R, Sakai K, Sugawara Y, et al. Case report: the operation for the lumbar disk herniation just after cesarean delivery in the third trimester of pregnancy. Int $\mathbf{J}$ Surg Case Rep. 2014;5:1178-82.

10. Choi BS, Choi I, Lee S. Surgical strategy using endoscopy to minimize fetal surgical risks in treating a pregnant woman with herniated lumbar disc disease. Indian J Neurosurg. 2016;5:051-4.

11. Bitterman J, Donovan J, Lam M. Diagnosis and rehabilitation of a pregnant woman with spinal cord disorder due to spinal cord tumor. Spinal Cord Ser Cases. 2019;5:8. 